\title{
Character Association and Path Coefficient Analysis in Bitter Gourd (Momordica charantia L.) Genotypes
}

\author{
H.M. Sowmya*, Shashikala S. Kolakar, D. Lakshmana, \\ Sadashiv Nadukeri, V. Srinivasa and Sridevi A. Jakkeral
}

Department of Crop Improvement and Biotechnology, College of Horticulture, Mudigere University of Agricultural and Horticultural Sciences, Shivamogga, India

*Corresponding author

\begin{tabular}{|c|c|}
\hline & A B S T R A C T \\
\hline $\begin{array}{l}\text { K e y w o r d s } \\
\text { Path coefficient, } \\
\text { Bitter gourd, Fruit } \\
\text { yield, Vine length }\end{array}$ & $\begin{array}{l}\text { Correlation and path coefficients analysis can helps to assess the mutual relationship } \\
\text { between various plant characters and determines component characters on which selection } \\
\text { can be based or improvement in yield. Twenty four genotypes of bitter gourd were } \\
\text { evaluated for yield contributing characters to observe their associations and direct and } \\
\text { indirect effect on fruit yield at College of Horticulture, Mudigere during summer 2017-18. }\end{array}$ \\
\hline Article Info & $\begin{array}{l}\text { The study revealed that genotypic correlation coefficient was higher than the respective } \\
\text { phenotypic correlation coefficients; this indicates the lesser influence on phenotypic }\end{array}$ \\
\hline $\begin{array}{l}\text { Accepted: } \\
17 \text { April } 2019 \\
\text { Available Online: } \\
10 \text { May } 2019\end{array}$ & $\begin{array}{l}\text { expression. Fruit yield per plant had significant positive correlation with fruit length and } \\
\text { fruit weight. High positive direct effect was observed between fruit yield per plant with } \\
\text { vine length, node at which male flower appears, number of fruits per vine, fruit weight and } \\
\text { fruit length which are important characters to be accounted for gaining improvement in } \\
\text { yield. }\end{array}$ \\
\hline
\end{tabular}

\section{Introduction}

Bitter gourd (Momordica charantia L.) is an important tropical and sub-tropical vine belongs to the family Cucurbitaceae. The genus derived its name from the Latin name, mordicus meaning bitten. Among different species Momordica charantia L. is widely cultivated species having chromosome number $2 \mathrm{n}=22$. It is a versatile, underutilized high-value vegetable in India having nutritional (Ojha et al., 2009) and medicinal importance (Choudhary, 1967). The improvement made in crop varieties is mainly concentrated on increasing yield and its attributing characters. A study of the correlation between different quantitative characters provides an idea of association of different characters. It could be effectively exploited to formulate the selection strategies for improving yield and quality (Kalloo, 1994). Path coefficient provides an effective means of entangling direct and indirect causes of association and measures the relative 
importance of each causal factor. Partitioning of total correlation into direct and indirect effects would be worthwhile for an effective selection program.

\section{Materials and Methods}

Twenty four bitter gourd genotypes including some released varieties were evaluated at College of Horticulture, Mudigere during summer 2017-18. The physically pure and healthy seeds of these genotypes were collected from different regions of Karnataka. Genotypes were studied using Randomized Block Design with three replications. Plants were grown at a spacing of $2 \mathrm{~m}$ between rows and $1.2 \mathrm{~m}$ between plants by adopting the package of practice, UHS, Bagalkot. Observations were recorded on five randomly selected plants of each genotype in each replication for thirteen characters, viz., Vine length (m), Number of branches per vine, Internodal length $(\mathrm{cm})$, Node at which first male flower appear, node at which first female flower appear, Days to first male flower, Days to first female flower, Sex ratio, Number of fruits per vine, Fruit weight $(\mathrm{g})$, Fruit length $(\mathrm{cm})$, Fruit width $(\mathrm{mm})$ and fruit yield per vine $(\mathrm{kg})$. Genotypic and phenotypic correlations were calculated as per Al-Jibouri et al., (1958) using an ANOVA and covariance matrix in which total variability was split into replications, genotypes, and errors. The genotypic and phenotypic correlation coefficients were used to determine direct and indirect contribution toward yield per plot. The direct and indirect paths were obtained according to the method of Dewey and Lu (1959).

\section{Results and Discussion}

Variability studies provide information on the extent of improvement in different characters, but not on the extent and nature of relationship existing between various contributory and economically important characters. The phenotypic and genotypic correlation studies were carried out to know the nature of relationship existing between yield and their component characters and are presented in the Tables 1 and 2 respectively.

\section{Phenotypic correlation}

Vine length had found significant and positive correlation with internodal length (0.41), fruit weight (0.27) and with node at which first female flower appear (0.26). Fruit yield per vine had highly significant and positive correlation with internodal length (0.48), number of fruits per vine (0.28) and node at which first female flower appear (0.26), internodal length had positive and non significant association with fruit yield per vine (0.05). Node at which first female flower appear (0.47) and node at which first male flower appear (0.39) showed highly significant and positive correlation with internodal length.

Node at which first male flower appears showed significant and positive association with node at which first female flower appears (0.74) and number of fruits per vine (0.23). Node at which first female flower appear showed significant positive correlation with number of fruits per vine (0.27), Fruit yield per vine (0.24) showed significant and positive association with number of fruits per vine and also the trait had significant and positive association with fruit weight (0.49) and fruit yield per vine (0.24). Fruit weight had highly significant and positive association with fruit yield per vine (0.70), fruit length (0.64) and fruit width (0.27) and fruit length had significant and positive correlation with fruit yield per vine (0.48). Similar observations were made by earlier workers Yadagiri et al., (2017) for number of fruits per vine, fruit length, Rani et al., (2015) for number of fruits per vine, average fruit 
weight, pulp thickness in bitter gourd, Khan et al., (2015) for fruit length, average fruit weight, number of fruits per vine in bitter gourd and Yadav and Yadav (2015) for average fruit weight only at phenotypic level, in bitter gourd.

Table.1 Phenotypic correlation coefficients among 12 yield and yield components in bitter gourd

\begin{tabular}{|c|c|c|c|c|c|c|c|c|c|c|c|c|c|}
\hline & $\mathbf{X}_{\mathbf{1}}$ & $\mathbf{X}_{\mathbf{2}}$ & $\mathbf{X}_{\mathbf{3}}$ & $\mathbf{X}_{\mathbf{4}}$ & $\mathbf{X}_{\mathbf{5}}$ & $\mathbf{X}_{\mathbf{6}}$ & $\mathbf{X}_{\mathbf{7}}$ & $\mathbf{X}_{\mathbf{8}}$ & $\mathbf{X}_{\mathbf{9}}$ & $\mathbf{X}_{\mathbf{1 0}}$ & $\mathbf{X}_{\mathbf{1 1}}$ & $\mathbf{X}_{\mathbf{1 2}}$ & $\mathbf{X}_{\mathbf{1 3}}$ \\
\hline $\mathbf{X}_{\mathbf{1}}$ & 1.00 & 0.22 & $0.41^{* *}$ & 0.06 & $0.26^{*}$ & $-0.38^{* *}$ & $-0.42^{* *}$ & 0.01 & -0.12 & $0.27^{*}$ & 0.19 & -0.01 & 0.19 \\
\hline $\mathbf{X}_{\mathbf{2}}$ & & 1.00 & $0.48^{* *}$ & 0.09 & $0.26^{*}$ & $-0.26^{*}$ & $-0.33^{* *}$ & $-0.29^{*}$ & $0.28^{*}$ & -0.17 & $-0.30^{* *}$ & 0.02 & -0.02 \\
\hline $\mathbf{X}_{\mathbf{3}}$ & & & 1.00 & $0.39^{* *}$ & $0.47^{* *}$ & $-0.32^{* *}$ & $-0.28^{*}$ & -0.20 & 0.17 & 0.04 & -0.06 & 0.01 & 0.05 \\
\hline $\mathbf{X}_{\mathbf{4}}$ & & & & 1.00 & $0.74^{* *}$ & -0.14 & -0.16 & -0.03 & $0.23^{*}$ & -0.19 & 0.18 & 0.02 & -0.05 \\
\hline $\mathbf{X}_{\mathbf{5}}$ & & & & 1.00 & -0.04 & -0.08 & 0.01 & $0.27^{*}$ & $-0.32^{* *}$ & 0.03 & $-0.23^{*}$ & -0.19 \\
\hline $\mathbf{X}_{\mathbf{6}}$ & & & & & & 1.00 & $0.83^{* *}$ & 0.15 & -0.07 & -0.01 & -0.07 & $-0.27^{*}$ & -0.10 \\
\hline $\mathbf{X}_{\mathbf{7}}$ & & & & & & 1.00 & -0.02 & 0.01 & -0.10 & -0.09 & $-0.35^{* *}$ & -0.14 \\
\hline $\mathbf{X}_{\mathbf{8}}$ & & & & & & & 1.00 & $-0.27^{*}$ & 0.05 & 0.19 & $-0.38^{* *}$ & -0.14 \\
\hline $\mathbf{X}_{\mathbf{9}}$ & & & & & & & & 1.00 & $0.49^{* *}$ & $-0.28^{*}$ & $-0.23^{*}$ & $0.24^{*}$ \\
\hline $\mathbf{X}_{\mathbf{1 0}}$ & & & & & & & & & & 1.00 & $0.64^{* *}$ & $0.27^{*}$ & $0.70^{* *}$ \\
\hline $\mathbf{X}_{\mathbf{1 1}}$ & & & & & & & & & & & 1.00 & 0.05 & $0.48^{* *}$ \\
\hline $\mathbf{X}_{\mathbf{1 2}}$ & & & & & & & & & & & & 1.00 & 0.14 \\
\hline
\end{tabular}

Critical $\mathrm{r}_{\mathrm{P}}$ value $=0.30$ at $1 \%$ and 0.23 at $5 \% \quad *$ Significant at $5 \%$ probability level, $\quad * *$ Significant at $1 \%$ probability level

Where,

$\mathrm{X}_{1}=$ Vine length $(\mathrm{m})$

$\mathrm{X}_{4}=$ Node at which first male flower appear

$\mathrm{X}_{7}=$ Days to first female flower

$\mathrm{X}_{2}=$ Number of branches per vine

$\mathrm{X}_{3}=$ Internodal length $(\mathrm{cm})$

$\mathrm{X}_{5}=$ Node at which first female flower appear $\quad \mathrm{X}_{6}=$ Days to first male flower

$\mathrm{X}_{10}=$ Fruit weight $(\mathrm{g})$

$\mathrm{X}_{8}=$ Sex ratio

$\mathrm{X}_{13}=$ Fruit yield per vine $(\mathrm{kg})$

$\mathrm{X}_{11}=$ Fruit length $(\mathrm{cm})$

$\mathrm{X}_{9}=$ Number of fruits per vine $\mathrm{X}_{12}=$ Fruit width $(\mathrm{mm})$

Table.2 Genotypic correlation coefficients among 12 yield and yield components in bitter gourd

\begin{tabular}{|c|c|c|c|c|c|c|c|c|c|c|c|c|c|}
\hline & $\mathbf{X}_{1}$ & $\mathbf{X}_{2}$ & $\mathbf{X}_{\mathbf{3}}$ & $\mathbf{X}_{4}$ & $\mathbf{X}_{\mathbf{5}}$ & $\mathbf{X}_{6}$ & $\mathbf{X}_{\mathbf{7}}$ & $\mathbf{X}_{8}$ & $\mathbf{X}_{9}$ & $\mathbf{X}_{10}$ & $\mathbf{X}_{11}$ & $X_{12}$ & $\mathbf{X}_{13}$ \\
\hline $\mathbf{X}_{1}$ & 1.00 & $0.25 *$ & $0.41 * *$ & 0.05 & $0.29 *$ & $-0.49 * *$ & $-0.56^{* *}$ & 0.01 & -0.12 & $0.29 *$ & $0.24 *$ & 0.01 & 0.19 \\
\hline $\mathbf{X}_{2}$ & & 1.00 & $0.57 * *$ & 0.08 & $0.32 * *$ & $-0.26^{*}$ & $-0.33 * *$ & $-0.32 * *$ & $0.34 * *$ & -0.17 & $-0.33 * *$ & 0.05 & -0.02 \\
\hline $\mathbf{X}_{3}$ & & & 1.00 & $0.45 * *$ & $0.51 * *$ & $-0.37 * *$ & $-0.34 * *$ & -0.22 & 0.17 & 0.04 & -0.05 & 0.01 & 0.05 \\
\hline $\mathbf{X}_{4}$ & & & & 1.00 & $0.80 * *$ & -0.15 & -0.15 & -0.04 & $0.26 *$ & -0.21 & 0.21 & 0.04 & -0.05 \\
\hline$X_{5}$ & & & & & 1.00 & -0.06 & -0.12 & -0.03 & $0.29 *$ & $-0.33^{* *}$ & 0.04 & $-0.24 *$ & -0.19 \\
\hline$X_{6}$ & & & & & & 1.00 & $0.92 * *$ & 0.15 & -0.08 & -0.03 & -0.05 & $-0.32 * *$ & -0.10 \\
\hline $\mathbf{X}_{7}$ & & & & & & & 1.00 & -0.04 & 0.01 & -0.12 & -0.09 & $-0.39 * *$ & -0.14 \\
\hline $\mathbf{X}_{8}$ & & & & & & & & 1.00 & $-0.28 *$ & 0.05 & 0.19 & $-0.41 * *$ & -0.14 \\
\hline$X_{9}$ & & & & & & & & & 1.00 & $0.50 * *$ & $-0.30 * *$ & $-0.25^{*}$ & $0.24 *$ \\
\hline $\mathbf{X}_{10}$ & & & & & & & & & & 1.00 & $0.67 * *$ & $0.27 *$ & $0.70 * *$ \\
\hline $\mathbf{X}_{11}$ & & & & & & & & & & & 1.00 & 0.05 & $0.48 * *$ \\
\hline$X_{12}$ & & & & & & & & & & & & 1.00 & 0.14 \\
\hline
\end{tabular}

Critical $r_{\mathrm{P}}$ value $=0.30$ at $1 \%$ and 0.23 at $5 \% \quad *$ Significant at $5 \%$ probability level, $* *$ Significant at $1 \%$ probability level

Where,

$\mathrm{X}_{1}=$ Vine length $(\mathrm{m})$

$\mathrm{X}_{4}=$ Node at which first male flower appear

$\mathrm{X}_{7}=$ Days to first female flower

$\mathrm{X}_{10}=$ Fruit weight $(\mathrm{g})$

$\mathrm{X}_{13}=$ Fruit yield per vine $(\mathrm{kg})$
$\mathrm{X}_{2}=$ Number of branches per vine $\quad \mathrm{X}_{3}=$ Internodal length $(\mathrm{cm})$

$\mathrm{X}_{5}=$ Node at which first female flower appear $\quad \mathrm{X}_{6}=$ Days to first male flower

$\mathrm{X}_{8}=$ Sex ratio

$\mathrm{X}_{11}=$ Fruit length $(\mathrm{cm})$

$$
\mathrm{X}_{12}=\text { Fruit width }(\mathrm{mm})
$$


Table.3 Path coefficients among yield and yield components in bitter gourd

\begin{tabular}{|l|l|l|l|l|l|l|l|l|l|l|l|l|l|}
\hline & $\mathbf{X}_{\mathbf{1}}$ & $\mathbf{X}_{\mathbf{2}}$ & $\mathbf{X}_{\mathbf{3}}$ & $\mathbf{X}_{\mathbf{4}}$ & $\mathbf{X}_{\mathbf{5}}$ & $\mathbf{X}_{\mathbf{6}}$ & $\mathbf{X}_{\mathbf{7}}$ & $\mathbf{X}_{\mathbf{8}}$ & $\mathbf{X}_{\mathbf{9}}$ & $\mathbf{X}_{\mathbf{1 0}}$ & $\mathbf{X}_{\mathbf{1 1}}$ & $\mathbf{X}_{\mathbf{1 2}}$ & $\mathbf{X}_{\mathbf{1 3}}$ \\
\hline $\mathbf{X}_{\mathbf{1}}$ & $\mathbf{0 . 0 4 1}$ & 0.009 & 0.016 & 0.002 & 0.010 & -0.015 & -0.017 & 0.004 & -0.005 & 0.011 & 0.008 & -0.001 & 0.192 \\
\hline $\mathbf{X}_{\mathbf{2}}$ & -0.001 & $\mathbf{- 0 . 0 0 7}$ & -0.003 & -0.001 & -0.002 & 0.002 & 0.002 & 0.002 & 0.002 & 0.001 & 0.002 & 0.000 & -0.021 \\
\hline $\mathbf{X}_{\mathbf{3}}$ & -0.076 & -0.090 & $\mathbf{- 0 . 1 8 7}$ & -0.072 & -0.087 & 0.059 & 0.052 & 0.037 & -0.032 & -0.008 & 0.012 & -0.002 & 0.051 \\
\hline $\mathbf{X}_{\mathbf{4}}$ & 0.006 & 0.009 & 0.040 & $\mathbf{0 . 1 0 2}$ & 0.075 & -0.015 & -0.017 & -0.003 & 0.023 & -0.020 & 0.019 & 0.002 & -0.049 \\
\hline $\mathbf{X}_{\mathbf{5}}$ & -0.009 & -0.010 & -0.018 & -0.028 & $\mathbf{- 0 . 0 3 8}$ & 0.002 & 0.003 & 0.000 & -0.010 & 0.012 & -0.001 & 0.009 & -0.192 \\
\hline $\mathbf{X}_{\mathbf{6}}$ & 0.027 & 0.018 & 0.022 & 0.010 & 0.003 & $\mathbf{- 0 . 0 7 1}$ & -0.058 & -0.010 & 0.005 & 0.001 & 0.005 & 0.019 & -0.104 \\
\hline $\mathbf{X}_{\mathbf{7}}$ & 0.008 & 0.006 & 0.005 & 0.003 & 0.002 & -0.015 & $\mathbf{- 0 . 0 1 9}$ & 0.000 & 0.000 & 0.002 & 0.002 & 0.007 & -0.139 \\
\hline $\mathbf{X}_{\mathbf{8}}$ & -0.001 & 0.016 & 0.011 & 0.001 & 0.000 & -0.008 & 0.000 & $\mathbf{- 0 . 0 5 6}$ & 0.015 & -0.003 & -0.012 & 0.021 & -0.144 \\
\hline $\mathbf{X}_{\mathbf{9}}$ & -0.079 & 0.189 & 0.115 & 0.153 & 0.182 & -0.046 & 0.005 & -0.179 & $\mathbf{0 . 6 7 3}$ & 0.330 & -0.186 & -0.158 & 0.243 \\
\hline $\mathbf{X}_{\mathbf{1 0}}$ & 0.289 & -0.180 & 0.045 & -0.209 & -0.346 & -0.013 & -0.113 & 0.059 & -0.534 & $\mathbf{0 . 8 9 3}$ & 0.697 & 0.289 & 0.701 \\
\hline $\mathbf{X}_{\mathbf{1 1}}$ & -0.012 & 0.019 & 0.004 & -0.012 & -0.002 & 0.004 & 0.006 & -0.012 & 0.017 & -0.040 & $\mathbf{- 0 . 0 6 3}$ & -0.003 & 0.480 \\
\hline $\mathbf{X}_{\mathbf{1 2}}$ & 0.004 & -0.001 & 0.000 & -0.001 & 0.010 & 0.012 & 0.016 & 0.017 & 0.011 & -0.012 & -0.002 & $-\mathbf{0 . 0 4 5}$ & 0.139 \\
\hline
\end{tabular}

Residual effect $=0.38$

Bold diagonal value indicates direct effect

Critical $\mathrm{r}_{\mathrm{P}}$ value $=0.302$ at 1 per cent and 0.232 at 5 per cent probability level

$\mathrm{r}_{\mathrm{p}}=$ Phenotypic path coefficients with fruit yield per vine $(\mathrm{kg})$

\section{Genotypic correlation}

The data pertaining to the genotypic correlation coefficients for different characters among bitter gourd genotypes are presented in Table 2. Vine length was positively and significantly correlated with internodal length (0.41), node at which first female flower appear (0.29), fruit weight (0.29), number of branches per vine (0.25) and fruit length $(0.24)$. Number of branches per vine had shown significant and positive correlation with internodal length (0.57), number of fruits per vine (0.34) and node at which first female flower appear (0.32). Internodal length exhibited highly significant and positive correlation with node at which first female flower appear (0.51) and node at which first male flower appear (0.45). Node at which first female flower appear showed significant and positive correlation with number of fruits per vine (0.29). Number of fruits per vine exhibited significant and positive correlation (0.24) with fruit yield per vine and fruit weight $(0.50)$.

\section{Path coefficient analysis}

Path coefficient analysis gives relative contribution of different characters towards the fruit yield per vine. By partitioning the correlation coefficient into direct and indirect effect of a selected trait on fruit yield per vine and its indirect effect through other characters were computed and presented in Table 3. Fruit yield per vine had direct positive effect via vine length (0.041), node at which first male flower appear (0.102), number of fruits per vine (0.673) and fruit weight (0.893). This indicates that there is strong association between these vegetative traits this results agrees with Rani et al., (2015) for internodal length, fruit weight and fruit length.

In conclusion, the traits viz., vine length, node at which male flower appears, number of fruits per vine, fruit weight and fruit length are important characters to be accounted for gaining improvement in fruit yield per vine. Since, these characters had high positive direct effects on fruit yield per vine. 


\section{References}

AL-Jibouri, H. A. Millar, P. A. and Robinson, H. F., Genotypic and environmental variances and co-variances in an upland cotton cross of interspecific origin. Agronomy Journal. 1958; 50:633-636.

Choudhary, B., 1967, Vegetable National Book Trust. New Delhi, India.

Dewey, O. R. and Lu, K. H., Correlation and path coefficient analysis of components of crested wheat grass seed production. Journal of Agronomy. 1959; 51:515518.

Kalloo, G., 1994, Vegetable breeding. Panima Educational Book Agency, 51.

Khan, M. H., Bhuiyan, S. R., Saha, K. C. M. R., Bhuyin, M. R. and Ali, A. S. M. Y., 2015, Variability correlation and path co-efficient analysis of bitter gourd (Momordica charantia L.). Bangladesh J. Agril. Res., 40(4): 607- 618.
Ojha, M. D., Pandey, V. S. and Singh, G., 2009, Heterosis and combining ability in bitter gourd of Asian bitter gourd (Momordica charantia L.). Australian J. Crop Sci., 6(2): 261-267.

Rani, K. R., Raju, S. and Reddy, K. R., 2015, Variability, correlation and path analysis in bitter gourd (Momordica charantia L.). Agric. Sci. Digest, 35(2): 106-110.

Yadagiri, J., Gupta, N. K., Tembhre, D. and Verma, S., 2017, Genetic variability, correlation studies and path coefficient analysis in bitter gourd (Momordica charantia L.). J. Pharmacogn. Phytochem., 6(2): 63-66.

Yadav, P. S. and Yadav, G. C., 2015, Genetic variability, correlation and pathcoefficient analysis in bitter gourd (Momordica charantia L.). Trends in biosciences, 8(4): 873-878.

\section{How to cite this article:}

Sowmya, H.M., Shashikala S. Kolakar, D. Lakshmana, Sadashiv Nadukeri, V. Srinivasa and Sridevi A. Jakkeral. 2019. Character Association and Path Coefficient Analysis in Bitter Gourd (Momordica charantia L.) Genotypes. Int.J.Curr.Microbiol.App.Sci. 8(05): 2193-2197. doi: https://doi.org/10.20546/ijcmas.2019.805.258 